\title{
Características de los cuidadores de enfermos mentales en Neiva, diciembre 2010 y enero 2011
}

\section{Characteristics of caregivers of mentally ill in Neiva, in december 2010 and january 2011}

Viviana Andrea Calderón Ramírez ${ }^{1}$

Palabras clave: enfermedad mental, características de los cuidadores.
Key words: mental illness, characteristics of caregivers.

\section{Resumen}

Objetivo: caracterizar al cuidador del paciente con trastorno mental, sus condiciones sociodemográficas, para reconocer sus carencias, necesidades y plantear la creación de redes especializadas de cuidadores a través de la cual se obtenga capacitación y financiación por parte del estado $u$ otro tipo de entidades interesadas.

Materiales y métodos: se realizó un estudio cuantitativo, descriptivo de corte transversal en el Hospital Universitario de Neiva entre diciembre de 2010 y enero de 2011 aplicando una encuesta a 40 pacientes hospitalizados y a 28 cuidadores. Se estudiaron las variables edad, sexo, estrato socioeconómico, ocupación, nivel educativo, estado civil, lugar de procedencia y nivel de ingresos económicos.

Resultados: la mayoría de los cuidadores están entre los 36 y 53 años, son de género femenino y proceden del departamento del Huila, viven en unión libre, vinculados al régimen subsidiado, pertenecen al estrato socioeconómico bajo, tienen estudios de secundaria incompleta, no poseen vínculo laboral en el momento de la entrevista y no cuentan con ingreso económico.

Conclusión: el estudio mostro la desprotección de enfermos y familiares cuidadores, por tal razón, existe la necesidad de la creación de una red de cuidadores a través de la cual se proteja, con la participación activa del estado, la familia, el usuario y las instituciones científicas, a los enfermos y a sus familias.
Abstract
Objective: With the purpose of knowing whether or not the mental health problem patient has a caregiver or not; and if it does, identify the caregivers' characteristics in order to know his socio-demographic conditions to get to his needs; to come out with legal mechanisms to form and specialized network with the intention of acquiring training and money from the state or other interested institution.
Materials and methods: a quantitative study was performed, descriptive of transversal kind at HUHMP from Neiva city, December 2010 to January 2011 surveying 40 hospitalized patients and 28 caregivers. It was have into account variables such as age, gender, socioeconomic strata, occupation, and level of education, civil state, and income.
Results: most of the caregivers average 35 to 53 years old, from the Huila department, living on welfare, low strata, and low education, unemployed and without income.
Conclusion: the study shows the abandonment of the mentally ill patient and caregivers by the governmental institutions, and, the need of creating a caregivers network to actively protect the family, patients, and caregivers.

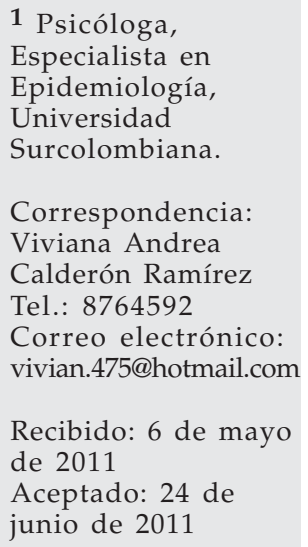

Recibido: 6 de mayo de 2011

Aceptado: 24 de junio de 2011 


\section{Introducción}

Los trastornos mentales y conductuales se consideran afecciones de importancia clínica, caracterizadas por alteraciones de los procesos de pensamiento, de la afectividad (emociones) o del comportamiento asociadas con angustia personal, alteraciones del funcionamiento o a ambos. No son sólo variaciones dentro de la "normalidad", sino fenómenos claramente anormales o patológicos. Para clasificarse como trastornos, estas anomalías deben ser duraderas o recurrentes y deben causar cierta angustia personal o alteraciones del funcionamiento en una o más facetas de la vida ${ }^{(1,14,15)}$.

En el mundo, cerca de 450 millones de personas están afectadas por un trastorno mental o de conducta. De acuerdo con la carga global de las enfermedades 2001 de la OMS, el 33\% de los años vividos con discapacidad son causados por los trastornos neuropsiquiátricos, solo el trastorno de depresión unipolar causa $12,15 \%$ de años vividos con discapacidad y constituye la tercera causa contribuyente a la carga global de las enfermedades. Cuatro de las seis causas más frecuentes de años vividos con discapacidad son la depresión, los trastornos debidos al uso de alcohol, la esquizofrenia y el trastorno bipolar. A las condiciones neuropsiquiátricas se les imputa el 13\% de los años de vida ajustados por discapacidad, a las lesiones autoinfligidas el 3,3\% y al VIH/SIDA otro $6 \%$. Estas dos últimas causas incluyen un componente conductual ${ }^{(1,14,15)}$.

Alrededor de 25 millones de personas sufren de esquizofrenia, 38 millones están afectadas por la epilepsia; más de 90 millones sufren de trastornos causados por el uso de alcohol y las drogas, y una de cada cuatro familias tiene por lo menos un miembro afectado por un trastorno mental; sin embargo, el presupuesto para salud mental de la mayoría de los países es inferior al 1\% del gasto total en salud. La relación entre carga de morbilidad y gasto en salud es claramente desproporcionada, más del $40 \%$ de los países no disponen de una política de salud mental, y en más del $30 \%$ no existe un programa dedicado a ella ${ }^{(2,16)}$. Lo preocupante es que el número de individuos con trastornos probablemente aumentará con el envejecimiento de las poblaciones, y como resultado de conflictos sociales ${ }^{(1)}$.

Los factores asociados a la prevalencia, la aparición y la evolución de los trastornos mentales y conductuales son la pobreza, el sexo, la edad, los conflictos y desastres, las enfermedades físicas graves y el entorno sociofamiliar $(2,3,16)$.

La relación entre pobreza y salud mental es compleja y multidimensional. La pobreza y las situaciones que conlleva, como el desempleo, la baja instrucción, las privaciones y la falta de un hogar, no sólo abundan en los países pobres, sino que afectan también a una minoría considerable en los países ricos. Entre las víctimas de la pobreza y las privaciones es mayor la prevalencia de trastornos mentales y del comportamiento, incluidos los asociados al abuso de sustancias psicotrópicas. Esta mayor prevalencia puede explicarse por la acumulación de causas de trastornos mentales entre los pobres, así como por la transición de los enfermos mentales hacia la pobreza ${ }^{(2,4,17,18)}$. El costo de los problemas de salud mental en países desarrollados se estima entre el 3\% y el $4 \%$ del Producto Nacional Bruto. Más aún, las enfermedades mentales cuestan a las economías nacionales varios miles de millones de dólares, tanto en términos de gastos incurridos directamente como en la pérdida de la productividad ${ }^{(16,17)}$. El costo promedio anual, incluyendo costos médicos, farmacéuticos y por discapacidad, para trabajadores afectados por depresión puede llegar a ser 4,2 veces más alto que el incurrido por un asegurado típico ${ }^{(1)}$.

Los trastornos mentales representan una gran proporción de los años de vida potencialmente perdidos por discapacidad (AVPPD), y se predice que esta carga aumentará significativamente (OMS, 2001b) en el futuro $^{(6)}$. Por eso una baja oferta de atención en salud mental conlleva a resultados pobres, recaídas que podrían evitarse y acciones escasas de rehabilitación ${ }^{(6,17-19)}$. Las ciencias sociales y biológicas han ofrecido información abundante acerca del papel que desempeñan los factores de riesgo y de protección en los mecanismos de desarrollo de los trastornos mentales y en el deterioro de la salud 
mental. Muchos de estos factores son susceptibles de modificación y representan objetivos potenciales para la aplicación de estrategias de prevención y de promoción. Se ha demostrado que las estrategias preventivas reducen los factores de riesgo, refuerzan los factores de protección, disminuyen los síntomas psiquiátricos y reducen el inicio de algunos trastornos mentales; además, estas estrategias también contribuyen a una mejoría de la salud mental y física, lo cual, a su vez, genera efectos beneficiosos, tanto sociales como económicos ${ }^{(3,16-19)}$.

La creación de redes $\mathrm{u}$ asociaciones de familiares o cuidadores de personas con trastorno mental es relevante porque la discapacidad asociada a los trastornos emocionales y del comportamiento es superior a la observada para las enfermedades crónicas (hipertensión, diabetes, artritis, enfermedades del corazón, etc.) y en este orden de ideas una persona con discapacidad de acuerdo al grado de severidad de la misma va a depender y a demandar cuidados de $\operatorname{otros}^{(7)}$. La importancia de estas organizaciones está en que a través de ellas los cuidadores o familiares pueden recibir información sobre la enfermedad, sobre los planes de tratamiento ${ }^{(6,14,15,18)}$, grupos entre iguales o grupos de ayuda mutua y estrategias necesarias para afrontar las dificultades que supone la convivencia con un familiar diagnosticado de TGM $^{*}$ pero además la defensa de los derechos e intereses del colectivo, educación a la comunidad, influir en los planificadores de políticas de Salud Mental, denunciar el estigma y la discriminación y la reivindicación de la mejora de los servicios ${ }^{(5,8)}$. El objetivo del presente trabajo es caracterizar al cuidador del enfermo mental para identificar las necesidades económicas, sociales, clínicas y del cuidado tanto del paciente como del cuidador para impulsar la creación de una red de cuidadores de personas con trastorno mental en la ciudad de Neiva.

\section{Materiales y métodos}

Se realizó un estudio cuantitativo, descriptivo, prospectivo de corte transversal.
Se diseñaron 2 encuestas, una para aplicarla al paciente y otra para el cuidador, acompañadas de un formato de datos básicos que fueron extraídos de la historia clínica de los pacientes de la Unidad de Salud Mental (USM), del HUHMP en el periodo de diciembre de 2010 a enero 2011.

El investigador fue la persona quien aplicó el instrumento durante los días de visitas que fueron los días lunes, miércoles, jueves, viernes, sábado y domingo en la Unidad de salud mental en el horario de 2:00 pm hasta las 4:00 pm cada semana durante los meses de diciembre 2010 y enero 2011 hasta que se alcanzó la aplicación de la encuesta en un total de 40 pacientes y 28 cuidadores con previo consentimiento informado tanto de los pacientes como de los cuidadores que aceptaron participar del estudio. Todos los pacientes se abordaron de forma personal y presencial a diferencia de los cuidadores que en algunos casos no se entrevistaron personalmente, entonces se opto por hacer la entrevista de forma telefónica. De esta manera se entrevistó de forma personal a 19 cuidadores y por medio telefónico a 9, sin embargo de los 28 cuidadores entrevistados todos tienen medio telefónico a través del cual contactarlos, en un $98 \%$ celular.

Los pacientes encuestados fueron elegidos de forma no probabilística, no representativa por conveniencia, esto hace referencia a que no hay tamaño de muestra porque no se va a comparar ni a inferir sobre el universo. Cuando hablamos de muestreo por conveniencia por definición nos indica que esta sesgado a entrevistar a quienes encuentre aptos para responder la encuesta. Durante el mes de diciembre de 2010 se atendieron en la Unidad a 51 pacientes hospitalizados y en el mes de enero 2011 a 60; que en total suman 111 pacientes de los cuales se encuestaron a 40 de ellos por cumplir con los criterios de inclusión. La cantidad de pacientes entrevistados pudo ser diferente sin embargo la población es poco cambiante es decir que 1 paciente dura varios días o semanas por lo tanto fue frecuente en cada día de visita encontrar a los mismos pacientes que estaban aptos y a los que no, sin embargo siem- 
pre se tuvieron en cuenta los criterios de inclusión que comprenden: pacientes hospitalizados de la unidad de salud mental, mayores de 18 años, que no estuvieran sedados al momento de la entrevista, que fueran considerados aptos por el psiquiatra para responder la encuesta y que firmaran el consentimiento escrito (la consideración de aptos por el psiquiatra se relaciona con el examen del estado mental que hace el especialista al paciente cada mañana durante los días que este permanece hospitalizado y a través del cual va evidenciando progreso o no del mismo, lo que a su vez representa el ajuste de las dosis de los medicamentos, suspensión o cambio de estos y la orden de alta). Como criterios de exclusión: pacientes menores de 18 años, que no fueran aptos para la entrevista o que estuvieran sedados. En cuanto a los cuidadores es de mencionar que fueron seleccionados por conveniencia, ya que el paciente era quien refería si tenía o no quien lo cuidara, luego de explicarle cuando podía categorizar a una persona como cuidador y cuando no, no obstante este es un elemento subjetivo en donde el paciente de acuerdo a su interpretación cultural responde, y daba los datos para contactar al cuidador pero además esta información se contrastaba con la obtenida por el formato de datos extraídos de la historia clínica y el cuidador decidía si participaba o no del estudio. En los criterios de inclusión del cuidador se tuvo en cuenta que la persona fuera mayor de 18 años, y que consintiera participar y en los criterios de exclusión: que el cuidador tuviera menos de 18 años y que no consintiera participar, es de aclarar que no se tuvo en cuenta específicamente una apreciación de la capacidad mental del cuidador cuando este tenía una edad superior a 54 años, sin embargo 5 de las personas que hacían parte de este rango se entrevistaron personalmente y 2 se contactaron telefónicamente, por lo cual el investigador da fe de coherencia en el discurso y estuvo en capacidad de identificar alguna inhabilidad o alteración de la capacidad mental durante la entrevista de los cuidadores de acuerdo a su experiencia profesional, ya que si esto se hubiera presentado, no se hubiera pasado por alto sino que se hubiera puesto dentro de las observaciones.

Es importante mencionar que dentro de la definición de cuidador se consideró que: es "aquella persona que asiste o cuida a otra afectada de cualquier tipo de discapacidad, minusvalía o incapacidad que le dificulta o impide el desarrollo normal de sus actividades vitales o de sus relaciones sociales"(20), donde los miembros de la familia son, con frecuencia, los cuidadores primarios de las personas con trastorno mental y como tal ellos proveen apoyo emocional e instrumental, y con no menor frecuencia también deben afrontar los costos financieros asociados con el tratamiento y la atención.

Dentro de las variables estudiadas a nivel del paciente se encuentran: edad, sexo, estado civil, seguridad social, nivel educativo, lugar de procedencia (Depto y municipio), zona rural o urbana, tiempo de residencia en la vivienda actual, ocupación, tipo de ocupación dependiente o independiente, nivel de ingresos mensuales por la ocupación que realiza además de las características clínicas como diagnóstico, tiempo que lleva con el trastorno, tiene cuidador sí o no, parentesco, número de cuidadores, tiempo transcurrido desde que esta persona lo cuida y para los pacientes que no tienen cuidador, manifestar las razones del porque esta situación.

A los cuidadores aparte de las características sociodemográficas ya mencionadas en el paciente, otras como nivel socioeconómico, nivel del sisben, subsidio de familias en acción u otro subsidio del estado. Como también condiciones habitacionales de la vivienda donde habita el cuidador en relación a si la vivienda es arrendada, familiar o propia, número de habitaciones, número de habitantes por vivienda, material predominante en paredes, pisos y techo, si cuenta con servicio básico de energía eléctrica, alcantarillado, acueducto; cuando cocina lo hace con leña, estufa eléctrica o gas. Además de características sobre el cuidado en cuanto a si el cuidador tiene conocimiento del desarrollo de le enfermedad, si el personal de salud le ha explicado en qué consiste el trastorno y sus síntomas, si cuida el 
paciente desde el diagnostico, si las demás personas que viven en la vivienda participan en el cuidado, las horas diarias de cuidado y si está de acuerdo con la creación de una red especializada de cuidadores de pacientes con trastorno mental.

La prueba piloto se realizó aplicando el instrumento a 2 pacientes y a 1 cuidador; con el objetivo de valorar la confiabilidad y validez de este, y para detectar los posibles sesgos de medición con el fin de corregirlos.

La tabulación y análisis de los datos se realizó con el programa Epi Info, de Centers for Disease Control and Prevention, Atlanta, EE.UU. Para cada variable se hizo un análisis univariado con medidas de frecuencia. Este estudio fue previamente analizado y aprobado por el Comité de Ética Institucional tanto de la Universidad Surcolombiana como del HUHMP de Neiva.

\section{Resultados}

La mayoría $(72,5 \%)$ de pacientes son del sexo masculino. El grupo etario más frecuente está entre los 18 y 35 años con el $62,5 \%$. La mayoría de los pacientes (55\%) son solteros. En cuanto al sistema de seguridad social un $17,5 \%$ son del régimen vinculado.
En relación a las entidades promotoras de salud un $12,5 \%$ pertenece a Sanidad militar. En cuanto al nivel de escolaridad es importante destacar que un alto porcentaje $(25 \%)$ no terminó la primaria. La mayoría $(97,5 \%)$ de los pacientes son del Departamento del Huila. En lo que corresponde al municipio de donde provienen se identifica que Neiva presenta el $65 \%$ de la población y el $87,2 \%$ son de la zona urbana.

De los pacientes el 22,5\% llevan menos de 1 año residiendo en la vivienda actual. La mayoría $(77,5 \%)$ de los pacientes en el momento de la entrevista responden que sí trabajan y dentro del tipo de trabajo que desempeña se encuentra con mayor frecuencia $(57,6 \%)$ la actividad laboral independiente, así como dentro de las ocupaciones más comunes encontramos con igual porcentaje (5\%) las actividades de carpintería y ebanistería, seguido de construcción, mecánica de motos y recolección de café, cebolla y frijol. Es de mencionar que del 32,5\% de los pacientes que no reciben ingresos el 10\% aunque trabaja no recibe remuneración económica sino que su trabajo es compensado con hospedaje y alimentación (Tabla 1). Dentro de los diagnósticos más frecuentes encontramos el trastorno afectivo bipolar con el 30\%, así mismo identificando el tiempo

Tabla 1. Características sociodemográficas de los pacientes con trastorno mental de la Unidad de Salud Mental del Hospital Universitario Hernando Moncaleano Perdomo de Neiva-Huila. Diciembre 2010 y enero 2011.

\begin{tabular}{lcc}
\hline $\begin{array}{l}\text { Características sociodemográficas } \\
\text { paciente }\end{array}$ & $\mathbf{n}$ & \% \\
\hline $\begin{array}{l}\text { Género } \\
\text { Femenino }\end{array}$ & 11 & 27,5 \\
Masculino & 29 & 72,5 \\
Total & 40 & 100 \\
& & \\
Edad & & \\
Entre 18 y 35 & 25 & 62,5 \\
Entre 36 y 53 & 11 & 27,5 \\
Igual o superior a 54 & 4 & 10 \\
Total & 40 & 100 \\
& & \\
Estado civil & 3 & 7,5 \\
Casado & 22 & 55 \\
Soltero & 9 & 22,5 \\
Separado & 6 & 15 \\
Unión libre & -- & -- \\
Viudo & 40 & 100 \\
Total & & \\
\hline
\end{tabular}

\begin{tabular}{lcc}
\hline $\begin{array}{l}\text { Características sociodemográficas } \\
\text { paciente }\end{array}$ & $\mathbf{n}$ & \% \\
\hline Sssgs & 6 & \\
Contributivo & 22 & 15 \\
Subsidiado & 5 & 12,5 \\
Especial & 7 & 17,5 \\
Vinculado & 40 & 100 \\
Total & & \\
& & \\
Nivel de escolaridad & 1 & 2,5 \\
Sin estudios & 10 & 25 \\
Primaria incompleta & 6 & 15 \\
Primaria completa & 13 & 32,5 \\
Secundaria incompleta & 8 & 20 \\
Secundaria completa & 2 & 5 \\
Estudios superiores & 40 & 100 \\
Total & & \\
Departamento de donde proviene & 1 & 2,5 \\
Cundinamarca & 39 & 97,5 \\
Huila & 40 & 100 \\
Total &
\end{tabular}


Continuación Tabla 1.

\begin{tabular}{|c|c|c|}
\hline $\begin{array}{l}\text { Características sociodemográficas } \\
\text { paciente }\end{array}$ & $\mathrm{n}$ & $\%$ \\
\hline \multicolumn{3}{|l|}{ Trabaja actualmente } \\
\hline $\mathrm{Si}$ & 31 & 77,5 \\
\hline No & 9 & 22,5 \\
\hline Total & 40 & 100 \\
\hline \multicolumn{3}{|l|}{ Tipo de trabajo } \\
\hline Ninguno & 9 & 22,5 \\
\hline Dependiente & 8 & 20 \\
\hline Independiente & 23 & 57,5 \\
\hline Total & 40 & 100 \\
\hline \multicolumn{3}{|l|}{ Ocupación } \\
\hline Ninguna & 9 & 22,5 \\
\hline Agricultura & 1 & 2,5 \\
\hline Carpintería y ebanistería & 2 & 5 \\
\hline Construcción & 2 & 5 \\
\hline Construcción y ventas & 1 & 2,5 \\
\hline Conteo de personas centro comercial & 1 & 2,5 \\
\hline Cuidador de carros y corta pasto & 1 & 2,5 \\
\hline Cortador de pasto & 1 & 2,5 \\
\hline Empleada domestica & 1 & 2,5 \\
\hline Fabricación de plástico & 1 & 2,5 \\
\hline Estilista & 1 & 2,5 \\
\hline Ingeniería electrónica & 1 & 2,5 \\
\hline Sellador de bolsas & 1 & 2,5 \\
\hline Mecánica de motos & 2 & 5 \\
\hline Mecánica de motos y carros & 1 & 2,5 \\
\hline Moto taxi & 1 & 2,5 \\
\hline $\begin{array}{l}\text { Oficios varios (lavar y planchar ropa, } \\
\text { platos y aseo general) }\end{array}$ & 5 & 12,5 \\
\hline Reciclaje & 1 & 2,5 \\
\hline Recolección de café, cebolla y frijol & 2 & 5 \\
\hline Ejercito (soldados profesionales) & 4 & 10 \\
\hline Ventas ambulantes & 1 & 2,5 \\
\hline Total & 40 & 100 \\
\hline \multicolumn{3}{|l|}{ Ingresos mensuales en pesos } \\
\hline No trabajan + Ningún ingreso & 13 & 32,5 \\
\hline Menos de 1 salario mínimo & 18 & 45 \\
\hline Igual o superior al mínimo & 9 & 22,5 \\
\hline Total & 40 & 100 \\
\hline
\end{tabular}

de cronicidad del trastorno mental se reconoce que el $57 \%$ de los pacientes lleva entre 1 y 5 años con la enfermedad. En el momento de la entrevista el $25 \%$ de la población manifiesta no tener cuidador y de este porcentaje el $12,5 \%$ de los pacientes expresan que no tiene cuidador porque nunca han tenido quien les brinde cuidado y protección.

Por otro lado el parentesco que se presenta con mayor frecuencia es el de madre con un porcentaje del $25 \%$. El $65 \%$ de los pacientes tiene entre 1 y 2 cuidadores, y del período de tiempo que lleva esta persona cuidándolo un $10 \%$ lleva entre 10 y 52 años de dedicación y cuidado (Tabla 2).

Un total de 28 cuidadores participaron en el estudio de los cuales la mayoría $(71,4 \%)$
Tabla 2. Características clínicas de los pacientes con trastorno mental de la Unidad de Salud Mental del Hospital Universitario Hernando Moncaleano Perdomo de Neiva-Huila. Diciembre 2010 y enero 2011.

\begin{tabular}{|c|c|c|}
\hline Características clínicas & $\mathbf{n}$ & $\%$ \\
\hline \multicolumn{3}{|l|}{ Diagnóstico } \\
\hline Esquizofrenia & 10 & 25 \\
\hline $\begin{array}{l}\text { Psicosis secundaria a consumo } \\
\text { de sustancias }\end{array}$ & 8 & 20 \\
\hline Psicoactivas Trastorno afectivo bipolar & 12 & 30 \\
\hline Otros & 10 & \\
\hline Total & 40 & 100 \\
\hline \multicolumn{3}{|l|}{ Cronicidad del trastorno en años } \\
\hline Menos de 1 año & 9 & 22,5 \\
\hline Entre 1 y 15 años & 23 & 57,5 \\
\hline Más de 15 años & 8 & 20 \\
\hline Total & 40 & 100 \\
\hline \multicolumn{3}{|l|}{ Tiene cuidador } \\
\hline $\mathrm{Si}$ & 30 & 75 \\
\hline No & 10 & 25 \\
\hline Total & 40 & 100 \\
\hline \multicolumn{3}{|l|}{ Parentesco } \\
\hline Ninguno & 10 & 25 \\
\hline Amiga & 1 & 2,5 \\
\hline Cónyuge & 7 & 17,5 \\
\hline Cuñada & 1 & 2,5 \\
\hline Hermana & 4 & 10 \\
\hline Hermano & 2 & 5 \\
\hline Hija & 1 & 2,5 \\
\hline Hijo & 1 & 2,5 \\
\hline Madre & 10 & 25 \\
\hline Padre & 3 & 7,5 \\
\hline Total & 40 & 100 \\
\hline \multicolumn{3}{|l|}{ Tiempo desde que esta persona lo cuida } \\
\hline Ninguno & 10 & 25 \\
\hline Menos de 10 años & 25 & 62,5 \\
\hline Entre 10 y 31 años & 2 & 5 \\
\hline Entre 32 y 52 años & 2 & 5 \\
\hline Igual o superior a 53 & 1 & 2,5 \\
\hline Total & 40 & 100 \\
\hline
\end{tabular}

son de sexo femenino. El grupo etario más frecuente está entre 36 y 53 años, lo esperado de acuerdo a la edad joven de los pacientes. En cuanto al estado civil el 39,2\% vive en unión libre. El $60,7 \%$ son del régimen subsidiado lo que manifiesta el bajo nivel de ingresos que estos poseen y la mayoría $(39,25)$ están afiliados a comfamiliar como entidad promotora de salud. Por otro lado, la mayoría $(28,6 \%)$ de los cuidadores presenta secundaria incompleta, todos son del Departamento del Huila y un 28,7 viven en otros municipios diferentes a Neiva. El $89,25 \%$ se encuentran en zona urbana y un 14,2\% lleva menos de 1 año habitando la vivienda actual.

En lo que corresponde al trabajo el 57,1\% de los cuidadores no trabaja, situación que 
empieza a explicarse a partir de la edad de los cuidadores. De los que trabajan un $32,1 \%$ desarrollan ocupaciones de tipo independiente y dentro de las ocupaciones encontramos con igual porcentaje (7\%) agricultura, aseo, oficios varios y recolección de cosechas lo que confirma el grado de informalidad y subempleo.

Dentro del nivel de ingresos se identifica que el $57,1 \%$ de la población de los cuidadores no obtiene ningún ingreso, esto se confirma a la vez cuando al momento de la entrevista el $96,4 \%$ manifiesta ser de un nivel socioeconómico bajo y el $75 \%$ pertenecen al nivel 1 del SISBEN. Además es importante mencionar que el $89,2 \%$ no recibe subsidios del estado.

En cuanto a las características habitacionales de la vivienda del cuidador del enfermo mental se identifica que el $35,7 \%$ se encuentra en vivienda arrendada. El 82,1\% tienen entre 1 y 3 habitaciones por vivienda, además el 50\% de las viviendas albergan menos de 5 personas. Las familias se mantienen con más de 2,9 personas por cuarto, cuando se toma en cuenta el número de cuartos en sus viviendas.

Las paredes de la vivienda son de material en el $86 \%$ de ellas, solo el $68 \%$ tienen piso de cemento, el 93\% tiene techo de zinc, el $100 \%$ de la población está conectado al sistema de alcantarillado, 96\% conectado a red eléctrica, alcantarillado y el $86 \%$ de la población tiene servicio de gas natural para cocinar (Tabla 3).

El 25\% de los cuidadores manifiestan que las demás personas que viven con el paciente no participan en el cuidado del mismo. El $60,7 \%$ de los cuidadores no tiene conocimiento sobre el desarrollo de la enfermedad, signos y síntomas y el $57,1 \%$ reconoce que no ha recibido alguna clase de orientación o explicación por parte del personal de salud sobre la enfermedad. Un $75 \%$ cuidan al paciente desde el diagnóstico de la enfermedad y el $64,2 \%$ le dedican al paciente más de 12 horas diarias al cuidado. Al 100\% de los cuidadores les gustaría que se conformara una red de cuidadores a través del cual se les brinde información sobre el curso de la enfermedad, tratamiento, actualizaciones, entre otros.

\section{Discusión}

Entre los diversos factores que influyen en el proceso de tratamiento, recuperación y rehabilitación psicosocial del individuo con trastorno mental es importante destacar el papel que tiene la familia y los cuidadores cuando participan en dicho proceso, ya que a través de diferentes investigaciones con un alto nivel de evidencia se ha confirmado que cuando el paciente cuenta con un buen apoyo familiar y éste a su vez recibe psicoeducación, se reduce el número de recaídas o reingresos siendo los efectos observables hasta después de 7 años de la intervención, además que se mejora la utilización de los recursos y la práctica clínica, entre otros ${ }^{(5,9)}$. A pesar de que no han encontrado asociación entre el género del

Tabla 3. Características sociodemográficas de los cuidadores del paciente con trastorno mental de la Unidad de Salud Mental del Hospital Universitario Hernando Moncaleano Perdomo de Neiva-Huila. Diciembre 2010 y enero 2011.

\begin{tabular}{|c|c|c|}
\hline Características sociodemográficas & $\mathbf{n}$ & $\%$ \\
\hline \multicolumn{3}{|l|}{ Género } \\
\hline Femenino & 20 & 71,4 \\
\hline Masculino & 8 & 28,6 \\
\hline Total & 28 & 100 \\
\hline \multicolumn{3}{|l|}{ Edad } \\
\hline Entre 18 y 35 & 9 & 32,1 \\
\hline Entre 36 y 53 & 12 & 42,8 \\
\hline Igual o superior a 54 & 7 & 25 \\
\hline Total & 28 & 100 \\
\hline \multicolumn{3}{|l|}{ Estado civil } \\
\hline Casado & 6 & 21,4 \\
\hline Soltero & 1 & 3,6 \\
\hline Separado & 7 & 25 \\
\hline Unión libre & 11 & 39,2 \\
\hline Viudo & 3 & 10,7 \\
\hline Total & 28 & 100 \\
\hline \multicolumn{3}{|l|}{ Sssgs } \\
\hline Contributivo & 5 & 17,9 \\
\hline Subsidiado & 17 & 60,7 \\
\hline Especial & 2 & 7,1 \\
\hline Vinculado & 4 & 14,2 \\
\hline Total & 28 & 100 \\
\hline \multicolumn{3}{|l|}{ Nivel de escolaridad } \\
\hline Sin estudios & 1 & 3,6 \\
\hline Primaria incompleta & 6 & 21,4 \\
\hline Primaria completa & 7 & 25 \\
\hline Secundaria incompleta & 8 & 28,6 \\
\hline Secundaria completa & 4 & 14,2 \\
\hline Estudios superiores & 2 & 7,1 \\
\hline Total & 28 & 100 \\
\hline
\end{tabular}


Continuación Tabla 3.

\begin{tabular}{|c|c|c|}
\hline Características sociodemográficas & $\mathbf{n}$ & $\%$ \\
\hline \multicolumn{3}{|l|}{ Municipio donde vive } \\
\hline Campoalegre & 1 & 3,6 \\
\hline Garzón & 3 & 10,7 \\
\hline Iquira & 1 & 3,6 \\
\hline Neiva & 20 & 71,4 \\
\hline Palermo & 1 & 3,6 \\
\hline Suaza & 1 & 3,6 \\
\hline Teruel & 1 & 3,6 \\
\hline Total & 28 & 100 \\
\hline \multicolumn{3}{|l|}{ Trabaja } \\
\hline $\mathrm{Si}$ & 12 & 42,9 \\
\hline No & 16 & 57,1 \\
\hline Total & 20 & 100 \\
\hline \multicolumn{3}{|l|}{ Tipo de trabajo } \\
\hline Ninguno & 16 & 57,1 \\
\hline Dependiente & 3 & 10,7 \\
\hline Independiente & 9 & 32,1 \\
\hline Total & 28 & 100 \\
\hline \multicolumn{3}{|l|}{ Ocupación } \\
\hline Ninguna & 16 & 57,1 \\
\hline Agricultura & 2 & 7,1 \\
\hline Aseo & 2 & 7,1 \\
\hline Asesoría comercial & 1 & 3,6 \\
\hline Ebanistería & 1 & 3,6 \\
\hline Oficios varios & 2 & 7,1 \\
\hline Panadería & 1 & 3,6 \\
\hline Recolección de cosechas & 1 & 3,6 \\
\hline Ventas ambulantes & 2 & 7,1 \\
\hline Total & 28 & 100 \\
\hline \multicolumn{3}{|l|}{ Ingresos mensuales } \\
\hline Ninguno & 16 & 57,1 \\
\hline Menos de 1 salario mínimo & 10 & 35,7 \\
\hline Igual o superior al mínimo & 2 & 7,1 \\
\hline Total & 28 & 100 \\
\hline \multicolumn{3}{|l|}{ Nivel socioeconómico bajo } \\
\hline $\mathrm{Si}$ & 27 & 96,4 \\
\hline No & 1 & 3,6 \\
\hline Total & 28 & 100 \\
\hline \multicolumn{3}{|l|}{ Nivel de sisben } \\
\hline Ninguno & 1 & 3,6 \\
\hline 1 & 21 & 75 \\
\hline 2 & 5 & 17,8 \\
\hline 3 & 1 & 3,6 \\
\hline Total & & 100 \\
\hline \multicolumn{3}{|l|}{ Subsidio de familias en acción } \\
\hline No aplica & 1 & 3,6 \\
\hline No & 21 & 75 \\
\hline $\mathrm{Si}$ & 6 & 21,4 \\
\hline Total & 28 & 100 \\
\hline \multicolumn{3}{|l|}{ Otro subsidio } \\
\hline No aplica & 1 & 3,6 \\
\hline No & 25 & 89,2 \\
\hline $\mathrm{Si}$ & 2 & 7,1 \\
\hline Total & 28 & 100 \\
\hline
\end{tabular}

paciente y la prevalencia o incidencia (Kessler et al. 1994) de esquizofrenia y trastorno afectivo bipolar ${ }^{(2,9)}$, este estudio encontró que los pacientes de sexo femenino desarrollaron con mayor frecuencia trastorno afectivo bipolar a diferencia de los hombres, y que los pacientes del sexo masculino presentan mayor prevalencia del diagnóstico de esquizofrenia paranoide en comparación con las mujeres; sin embargo, la esquizofrenia parece tener un comienzo más temprano y una evolución más discapacitante en la población masculina (Sartorius et al. 1986)(2).

En cuanto al sistema de seguridad social menos de la cuarta parte de los pacientes son del régimen vinculado, siendo este un alto porcentaje que no se esperaría de acuerdo a las políticas de ampliación de cobertura y carnetización del sistema actual de salud. Con relación a las EPS llama la atención que un número significativo de personas pertenece a sanidad militar, esto cobra relevancia cuando la literatura señala que los desastres naturales, la violencia y la guerra traen consecuencias que alteran la salud mental del individuo y de las comunida$\operatorname{des}^{(2,6,3)}$. En cuanto al nivel de escolaridad es importante destacar que un alto porcentaje no terminó la primaria, varios estudios han demostrado una asociación significativa entre la prevalencia de "trastornos mentales comunes" y el bajo nivel educacional (Patel y Kleinman, 2003). Además se afirma que "El analfabetismo o el escaso nivel educacional y la enfermedad están íntimamente ligados en el ámbito de la pobreza"(2).

Es de mencionar que de los pacientes que no reciben ingresos, el $10 \%$ son personas que trabajan pero que aun así no reciben ingresos porque en lugar de remuneración económica son compensados con hospedaje y alimentación. Esta situación de inequidad e informalidad son las que demuestran el grado de discriminación y estigmatización en el que se encuentran los pacientes y esto a su vez, se relaciona con la pobreza(2); se ha demostrado que la pobreza simultáneamente se relaciona con el aumento de los síntomas psiquiátricos y en la morbilidad de los trastornos mentales ${ }^{(3)}$. Es fundamental resaltar que esto se complementa con la alta frecuencia de la actividad laboral indepen- 
diente, lo que pone de relieve la falta de oportunidades laborales dignas para esta pobla-

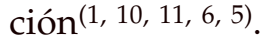

Se identificó que el diagnóstico con mayor frecuencia en el periodo de menos de 1 año de cronicidad es la esquizofrenia paranoide y en el periodo que comprende más de 15 años es el trastorno afectivo bipolar. Estos hallazgos eran de esperarse puesto estos dos trastornos son catalogados por las mismas características de su duración, síntomas y discapacidad en trastornos mentales graves ${ }^{(5)}$. Según la Guía de Práctica Clínica de intervenciones psicosociales en el trastorno mental grave "Parece que existe un consenso general de que el TMG puede afectar de manera considerable las relaciones en las familias de las personas que sufren la enfermedad, y que las relaciones familiares también pueden afectar de algún modo el curso de la enfermedad".

De acuerdo a las características del cuidador, los hallazgos confirman lo expuesto en la literatura acerca de las similitudes que presentan los cuidadores en cuanto a género, edad y parentesco ${ }^{(12,13)}$. Un número significativo de cuidadores presenta secundaria incompleta en su nivel de escolaridad.

Además es preocupante ver que varios de los cuidadores lleva entre 10 y 52 años de dedicación y cuidado al paciente, ya que esto evidencia el grado de discapacidad ${ }^{(1)}$ y cronicidad que genera la enfermedad mental pero además crea una carga de gran magnitud la cual afecta la calidad de vida familiar $^{(1)}$ tal como lo plantea la OMS cuando menciona que "la familia al tener un miembro afectado, está expuesta al estigma y discriminación como también al aislamiento, rechazo e imposibilidad de participar en redes sociales normales".

La mayoría de cuidadores son del régimen subsidiado y no obtiene ningún ingreso lo que resalta los pocos recursos económicos con los que cuentan y así mismo el estilo y la calidad de vida que llevan, esto se confirma cuando al momento de la entrevista la mayoría manifestó ser de un nivel socioeconómico bajo y pertenecer al nivel 1 del Sisben. Un gran porcentaje no recibe subsidios del estado lo que pone de relieve la falta de políticas claras frente a la priorización de subsidios a personas que cuidan o tienen un ser querido o familiar con enfermedad mental teniendo en cuenta el alto grado de improductividad que el trastorno le genera al paciente como también al cuidador ${ }^{(1)}$.

Solo menos de dos cuartas partes de los cuidadores reconoce que ha recibido alguna clase de orientación o explicación por parte del personal de salud sobre la enfermedad, cuando una amplia lista de estudios ha señalado que "las intervenciones psicoeducativas a los cuidadores han demostrado una mejora significativa en la carga que soportan los cuidadores y en la satisfacción percibida por el propio cuidador"(3). Es de resaltar que la mayoría de cuidadores cuidan al paciente desde el diagnóstico de la enfermedad y gran parte le dedican al paciente más de 12 horas diarias al cuidado.

Es posible afirmar que tanto los pacientes como los cuidadores comparten condiciones de pobreza, insalubridad, dificultades de vivienda, dificultades en acceso a salud, dificultades laborales (Arboleda-Flórez, $2001)^{(6)}$ entre otras, propias de las diferencias sociales. Además, es la familia o el cuidador quien dedica tiempo representativo para la atención del paciente afectado quienes por la ausencia de esquemas laborales especiales para enfrentar el problema se les torna difícil conseguir empleo o mantener el empleo actual, o se arriesgan a perder ingresos por ausencias forzadas del trabajo ${ }^{(1)}$.

Es común encontrar tal como lo menciona el Manual de Recursos de la OMS, que las familias y las personas a cargo asuman muchas responsabilidades vinculadas con el cuidado de las personas con trastornos mentales: entre ellas, su alojamiento, vestido y alimentación, y la de asegurarse de que recuerden seguir su tratamiento. También se aseguran que las personas con trastornos mentales, aprovechen los programas de atención y rehabilitación, y las ayudan a seguirlos. Con frecuencia deben soportar el lado adverso de la conducta de la persona cuando ésta se enferma o sufre una recaída, y son habitualmente los miembros de la familia y las personas a cargo quienes brin- 
dan amor, atención y se preocupan por la persona con trastornos mentales ${ }^{(1)}$.

Se hace indispensable que los miembros de la familia o cuidadores intervengan de forma organizada a través de redes especializadas (Ministerio de Sanidad y Política Social, 2009) para exigir y reclamar los derechos y el respeto que esta población merece en todos los aspectos que se encuentran alterados y deben empoderarse y contribuir en la formulación de políticas de salud mental, participar en el plan de tratamiento, especialmente cuando el paciente no está en condiciones de hacerlo solo (Ley de Atención en Salud Mental, Ley 24 de 1998, Isla Mauricio $)^{(1)}$, promover en la legislación la protección de las personas con trastornos mentales frente a la discriminación y a la explotación en el empleo ${ }^{(11)}$ ya que es el Estado quien debe hacerse cargo de la dependencia y vulnerabilidad que genera una situación de trastorno mental ${ }^{(10)}$.

\section{Conclusiones}

Los cuidadores de los enfermos mentales hospitalizados en la unidad de salud mental del HUHMP de Neiva, se caracterizan por ser de sexo femenino, madres de familia en edades entre 36 y 53 años, de estrato socioeconómico bajo, sin empleo e ingresos y viven en Neiva. Es importante mencionar que estos cuidadores no recibieron explicación $\mathrm{u}$ orientación por parte del personal de salud sobre la enfermedad, no conocen el desarrollo del trastorno, cuidan al paciente desde el diagnóstico; es decir, desde hace menos de 10 años y dedican más de 12 horas diarias de cuidado al paciente.

Es necesario crear una red de cuidadores a través de la cual se suministre ayuda mutua a sus participantes evitando la estigmatización y discriminación, obteniendo financiación que les permita brindar auxilios o subsidios a los cuidadores de los pacientes y a los pacientes para que cuenten con el recurso necesario para el transporte y los medicamentos, permitiendo así una mayor asistencia a los controles y una mejor adherencia al tratamiento, además de la realización de confe- rencias, talleres informativos sobre organización, grupos de apoyo, asistencia de relevo, capacitación a los cuidadores y a los profesionales y generando presión frente al gobierno para que aumente el presupuesto para la salud mental y desarrolle nuevas políticas de cuidado de la salud.

\section{Agradecimientos}

El autor expresa sus agradecimientos de manera especial al Doctor Rodrigo Alberto Álzate por asesorar el diseño, ejecución y análisis del presente estudio y porque siempre estuvo atento para aclarar cualquier duda o inquietud y se destacó por su profesionalismo y sentido humano.

\section{Referencias Bibliográficas}

1. Organización Mundial de la Salud. 2004. Invertir en salud. Departamento de Salud Mental y Abuso de Sustancias. Página web:http:/ / www.who.int/mental_health

2. Organización Mundial de la Salud. Informe sobre salud en el mundo 2001. Salud mental: nuevos conocimientos, nuevas esperanzas. Ginebra, Suiza.

3. Saxena, Sherhar. Llopis, Eva Jané. Hosman, Clemen. 2006. Prevención de los trastornos mentales y del comportamiento: implicaciones para la política sanitaria y la práctica clínica. World Psychiatry edición en español. Revista oficial de la asociación mundial de psiquiatría. 4(1): (WPA) Abril.

4. Londoño Nora H.; Marín Carlos A.; Juárez Fernando y otros. 2010. Factores de riesgo psicosociales y ambientales asociados a trastornos mentales. Suma psicológica, 17(1):7. Fundación Universitaria Konrad Lorenz, Colombia. Disponible en: http://redalyc. uaemex.mx/src/inicio/ArtPdfRed.jsp?i Cve $=134215244005$

5. Guía de Práctica Clínica de intervenciones psicosociales en el trastorno mental grave. Plan de Calidad para el Sistema Nacional de Salud del Ministerio de Sanidad y Política Social. Instituto Aragonés de Ciencias de la Salud-I+CS; 2009. Guías de Práctica Clínica en el SNS: I+CS N N $^{\circ} 2007 / 05$.

6. Organización Mundial de la Salud. Manual de recursos de la OMS sobre salud mental, derechos humanos y legislación. 2006. Ginebra, Suiza. 
7. Sandoval Mejía Sandra, Torres Mary Luz. Estudio epidemiológico de los trastornos mentales diagnosticados con mayor frecuencia por consulta externa y urgencias en el Centro de Rehabilitación Integral de Boyacá en los años 2003, 2004, 2005 y 2006. Tunja 2007. Consultado (27 de abril de 2011) Disponible en: http:/ / cribsaludmental. gov.co/morbilidad. htm\#cext

8. López Marcelino. 2004. Alternativas comunitarias ante el estigma y la discriminación. La experiencia de Andalucía España. Acta psiquiát psicol Am Lat 50(2):99-109.

9. Gutiérrez Achury A, Vanegas Vidal M, y otros. 2009. Factores farmacológicos y no farmacológicos que afectan la adherencia al tratamiento de los pacientes esquizofrénicos dados de alta de la unidad de salud mental del Hospital Universitario de Neiva entre enero y junio de 2008. Revista Facultad de Salud RFS julio-diciembre 2009. Universidad Surcolombiana. Neiva-Huila 1(2):25-30.

10. Seosane, Jose Antonio. 2006. Derecho y salud mental. Capacidades, derecho, justicia. Cuadernos de psiquiatría comunitaria. Tratamiento ambulatorio involuntario. 6(1).

11. Álvares López Marcelino. 2007. XII Jornadas de salud mental y justicia. Una relación compleja e imprescindible para la atención sanitaria y social a personas con trastornos mentales graves. Aracena 4-5 de octubre.

12. Espin Andrade, Ana Margarita. 2008. Caracterización psicosocial de cuidadores informales de adultos mayores con demencia. Rev Cubana Salud Pública [online]. 34(3): [citado 2010-06-28], pp. 0-0 . Disponible en: <http:/ /scielo.sld.cu/scielo.php?script $=$ sci_arttext \& pid = S0864-34662008000300008 $\& \operatorname{lng}=\mathrm{es} \& n r m=\mathrm{iso}>$. ISSN 0864-3466.

13. Burgos Garrido, Paola. Figueroa Rodríguez, Viviana, y otros. Caracterización y nivel de conocimiento del cuidador informal de usua- rios con dependencia severa adscritos al centro de salud Violeta Parra-Chillán Theoria [en línea] 2008, 17 (Sin mes). Consulta: (28 de junio de 2010) Disponible en: <http://redalyc.uaemex.mx/src/inicio/ ArtPdfRed.jsp?iCve $=29911533002>$

14. Organización Mundial de la Salud. 2004. Promoción de la salud mental. Conceptos, evidencia emergente práctica. Informe compendiado.

15. Organización Mundial de la Salud. 2004. Prevención de los trastornos mentales. Intervenciones efectivas y opciones de políticas. Informe compendiado.

16. Publicación oficial de la Federación Española de Asociaciones de Rehabilitación Psicosocial. 2007. Rehabilitación Psicosocial (fearp). Barcelona 2007 editor: Elsevier Doyma, S.L. Reservados todos los derechos. ISSN: 16969936 www.fearp.org 4(1).

17. López, Marcelino. 2004. Alternativas comunitarias ante el estigma y la discriminación. La experiencia de Andalucía España. Acta psiquiát psicol Am Lat 50(2):99-109.

18. Organización Mundial de la Salud. 2003. Mejora de la calidad de la salud mental. Conjunto de guías sobre servicios y politicas de salud mental.

19. Organización Mundial de la Salud. 2003. Legislación sobre salud mental y derechos humanos. Conjunto de guías sobre servicios y politicas de salud mental.

20. De los Reyes, María Cristina. Construyendo el concepto cuidador de ancianos. En: IV Reunión de Antropologia do Mercosul Foro de Investigación: Envejecimiento de la población en el Mercosur Noviembre. 2001. Curitiba. Brasil Consultado: (28 de junio 2010) Disponible en internet: http:// www.redadultosmayores.com.ar/docsPDF / Regiones/Mercosur/Brasil/06 Cuidadores deAncianos.pdf 
Universidad

Surcolombiana

\title{
GRUPO DE INVESTIGACIÓN, FACULTAD DE SALUD UNIVERSIDAD SURCOLOMBIANA
}

\author{
- BIOLOGÍA DE LA REPRODUCCIÓN \\ Líneas de investigación \\ 1. Biología Prostática \\ 2. Disruptores Endocrinos \\ 3. Cáncer \\ Página web: http://www.usco.edu.co/pagina/facultad-de-salud-grupos-y-semilleros-de- \\ investigacion \\ E-mail: garcia@usco.edu.co
}

\section{- PARASITOLOGÍA Y MEDICINA TROPICAL}

Líneas de investigación

1. Dengue

2. Entomología Médica

3. Infecciones recurrentes pediátricas

4. Parasitología Molecular

5. Epidemiologia y enfermedades infecciosas tropicales

Página web: http://portal.usco.edu.co/gruin/grupamed/

E-mail: jrodriguez@usco.edu.co

\section{- DNEUROPSY}

Líneas de investigación

1. Neurociencias, Procesos Neuropsicologicos Basicos

Página web: http://paginas.usco.edu.co/gruin/dneuropsy/

E-mail: mariapiedad@usco.edu.co

- DESARROLLO SOCIAL, SALUd PÚBLICA Y DERECHOS HUMANOS

Líneas de investigación

1. Desarrollo social, salud pública, y derechos humanos

2. Medicina de emergencias, cuidado crítico y reanimación

Página web: http://paginas.usco.edu.co/gruin/desarrollosocial/

E-mail: grupodsspdh@gmail.com

\section{- CARLOS FINLAY}

Líneas de investigación

1. El Suicidio en el Departamento del Huila

2. Morbilidad y mortalidad asociada a problemas de salud por causa externa

3. Salud pública

Página web: http://paginas.usco.edu.co/gruin/carlosfinlay/

E-mail:ninugo@usco.edu.co 